\title{
Reliability evaluation of distribution network based on improved non sequential Monte Carlo method
}

\author{
Jie Zhu' ${ }^{1,}$, Chao Li ${ }^{1, b}$, Aihong Tang ${ }^{1, c}$ \\ ${ }^{1}$ School of Automation, Wuhan University of technology, Wuhan, Hubei, 430070, China \\ a605281595@qq.com, b10136109@qq.com, c $375943243 @ q q . c o m$
}

Keywords: Reliability evaluation; Non sequential Monte Carlo sampling; Stratified sampling; Power system; Distribution network.

Abstract. In this paper the idea of stratification is utilized to form a homogeneous-stratified sampling method, which can increase the efficient of power system random fault state evaluation. Applying this method for reliability evaluation only generates a small number of independent random numbers, it decrease the amount of calculation and increase the efficient. The improved non sequential Monte Carlo method is then demonstrated and implemented in the reliability evaluation of RBTS-BUS5 system case, illustrating this algorithm can be used in reliability evaluation of distribution network.

\section{Introductions}

In order to obtain the reliability index of higher precision, it often requires a long calculation time. In order to reduce the simulation time and improve the convergence speed of Monte Carlo method, we can use the method of variance reduction. The usual methods of variance reduction are stratified sampling method, importance sampling method, the variable control method and dual variable method[1-4] and so on. However, there are some restrictive conditions of these methods in use.

In this paper, we use scattered sampling technique to reduce the variance, different from other traditional sampling method where is sampling interval uniform segmentation, take each sampling interval mean value of experiment function as the new experimental function of the system in every sampling, so as to reduce the simulation variance, improve simulation accuracy. The method can greatly reduce the sampling number does not exist any constraints, and therefore have a very good adaptability.

\section{The basic principle of non sequential Monte Carlo method}

The non sequential Monte Carlo simulation method [5] is often referred to as the state sampling method. This method is based on the system state is a combination of all elements, and each state of the element is determined by sampling probability of components in the state. Each element is available to be simulated by a uniform distribution in the interval of $[0,1]$. Assume that each element has two states of failure and work, and failure of components are independent each other. Let $s_{i}$ denote the state element $i, Q_{i}$ on behalf of the failure probability, generating a uniform random number $\mathrm{R}_{i}$ in the interval of $[0,1]$ for the element $i$, make 


$$
S_{i}=\left\{\begin{array}{lll}
0 & (\text { work state }) & \text { if } \mathrm{R}_{i}>Q_{i} \\
1 & (\text { failure state }) & \text { if } 0 \leq \mathrm{R}_{i} \leq Q_{i}
\end{array}\right.
$$

The system state with $\mathrm{N}$ elements is represented by the vector s:

$s=\left(\mathrm{s}_{1}, \ldots, \mathrm{s}_{i}, \ldots \mathrm{s}_{N}\right)$

When a system state is selected in the sample, namely carries on analyzing the system to determine whether it is a fault condition, if it is, then estimate risk index functions of the state. When the number of samples is large enough, the frequency of sampling system of S may be regarded as the probability of the unbiased estimation, i.e.

$$
P(\mathrm{~s})=\frac{m(\mathrm{~s})}{M}
$$

Where: $\mathrm{M}$ is the sampling number, $m(s)$ is the number of times of $\mathrm{S}$ appeared in the sampling system state.

\section{Sampling principle of distributed sampling method and its implementation process [6]}

The traditional sampling method is directly generated uniformly distributed random numbers in the interval of $[0,1]$, and determine the system stochastic state by the random number. But scattered sampling algorithm divided interval $[0,1]$ into $\mathrm{n}$ sub intervals. And the lengths of sub intervals meet $1 / n \geq \max \left\{\mathrm{f}_{1}, \mathrm{f}_{2}, \ldots \mathrm{f}_{m}\right\}$, where, $\mathrm{f}_{1}, \mathrm{f}_{2}, \ldots \mathrm{f}_{m}$ are the forced failure probability of system components. For a random number $x_{i}$, it can produce $\mathrm{n}$ state variables $\left(\mathrm{X}_{i 1}, \mathrm{X}_{i 2}, \ldots \mathrm{X}_{i j}, \ldots \mathrm{X}_{i n}\right)$ in the $\mathrm{n}$ intervals. In the $\mathrm{j}$ sub interval $\mathrm{X}_{i j}$ is determined according to the type 3

$$
\mathrm{X}_{i j}= \begin{cases}0 & \frac{j-1}{n} \leq x_{i}<\frac{j-1}{n}+f_{i} \\ 1 & x_{i}<\frac{j-1}{n} \text { or } x_{i} \geq \frac{j-1}{n}+f_{i}\end{cases}
$$

By the above formula indicates, when a random number vector $\mathrm{X}$ was generated, we can obtain $\mathrm{n}$ state vectors $\mathrm{X}_{i 1}, \mathrm{X}_{i 2}, \ldots \mathrm{X}_{i j}, \ldots \mathrm{X}_{i n}$ through the use of scattered sampling technique, it greatly improve the efficiency in the use of random number. Take the arithmetic average value of experimental function $F\left(\mathrm{X}_{1}\right), F\left(\mathrm{X}_{2}\right), \ldots F\left(\mathrm{X}_{i}\right), \ldots F\left(\mathrm{X}_{n}\right)$ in each sub interval as a random function of vector $\mathrm{X}$

$F(\mathrm{X})=\frac{1}{n} \sum_{j=1}^{n} F\left(\mathrm{X}_{j}\right)$

In order to realize the scattered sampling algorithm, we can use the calculated risk index the loss of load probability LOLP and the expected power not supplied value EPNS to achieve, in the piecewise interval $[j-1 / n, j / n]$, The experimental function is defined as follows:

When computing LOLP, experimental function as follows:

$$
F\left(\mathrm{X}_{j}\right)=\left\{\begin{array}{l}
1 \\
0 \quad \text { load cutting system } \\
0 \quad \text { load cutting system }
\end{array}\right.
$$

When computing EPNS, experimental function as follows: 


$$
F\left(\mathrm{X}_{j}\right)= \begin{cases}\sum_{i} P_{i} & \text { load cutting system } \\ 0 & \text { No load cutting system }\end{cases}
$$

Among them, $P_{i}$ is the value of load minus the total power outputting.

The new experimental function is:

$$
F^{*}(X)=\sum_{j=1}^{k} \frac{F\left(\mathrm{X}_{j}\right)}{n}
$$

The expected value formula of $F_{i}^{*}(\mathrm{X})$ is:

$$
E\left(F^{*}\right)=\sum_{i=1}^{N} \frac{F_{i}^{*}(\mathrm{X})}{N}
$$

In the formula above, $F_{i}^{*}(\mathrm{X})$ is the system ith sampling experiment function.

The variance estimation formula of $F^{*}(\mathrm{X})$ is:

$$
\mathrm{V}\left(F^{*}\right)=\sum_{i=1}^{N} \frac{\left[F_{i}^{*}(\mathrm{X})-E\left(F^{*}\right)\right]^{2}}{N}
$$

\section{Application of distributed sampling method in the reliability evaluation of distribution network}

Use the method above to evaluate the reliability for RBTS-BUS5 line of IEEE reliability testing system. RBTS-BUS 5 is a typical rural power distribution network as shown in Fig. 1. Table 1 and Table 2 separately listed the appropriate line data and user data. The rest data values were considered as: the value of line failure rate $\gamma_{L}$ is $0.2 /$ tim $e \times(\mathrm{km} \times \mathrm{a})^{-1}$, the value of repair time $\eta_{L}$ is $1.5 / h$ [7].

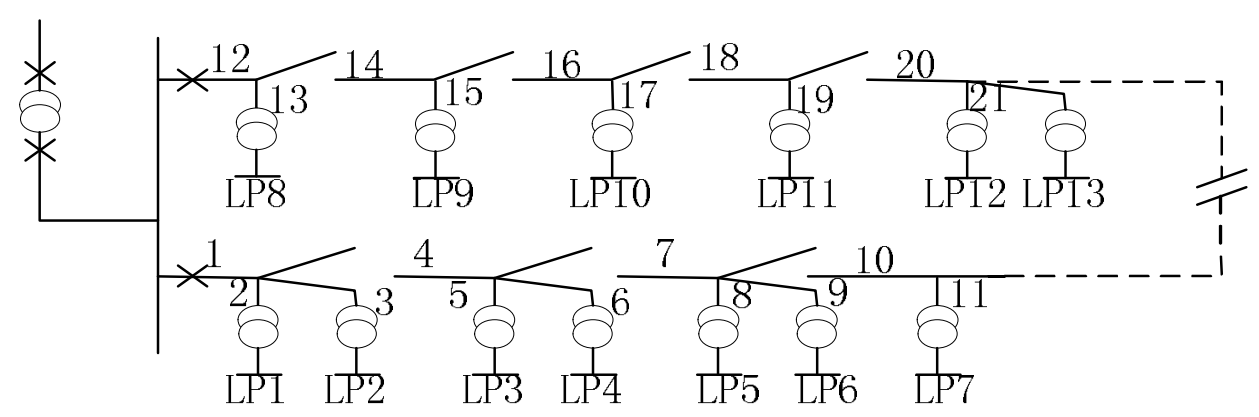

Fig.1 RBTS - BUS 5 The structure of ring network

Table 1 .The line data of RBTS-BUS5 ring network

\begin{tabular}{cc}
\hline Line number & Length $(\mathrm{km})$ \\
\hline $1,6,9,13,14,18,21$ & 0.5 \\
$4,7,8,12,15,16,19,22$ & 0.7 \\
$2,3,5,10,11,17,20$ & 0.8 \\
\hline
\end{tabular}


Table 2 .The user data of RBTS-BUS5 ring network

\begin{tabular}{ccccccc}
\hline Load point & Category & $\lambda /$ time $\cdot \alpha$ & $\mathrm{r} / \mathrm{h} \cdot$ time $^{-1}$ & $\begin{array}{c}\text { maximum } \\
\text { load }(\mathrm{kW})\end{array}$ & $\begin{array}{c}\text { average } \\
\text { load }(\mathrm{kW})\end{array}$ & $\begin{array}{c}\text { number } \\
\text { of users }\end{array}$ \\
\hline $1,2,3,4$ & Resident & 0.2360 & 1.5 & 740.0 & 0.4089 & 220 \\
$5,6,7$ & Agriculture & 0.2165 & 1.6 & 752.5 & 0.4169 & 1 \\
$8,9,10,11$ & Resident & 0.2588 & 1.4 & 1110.0 & 0.6247 & 1 \\
12,13 & Agriculture & 0.2263 & 1.5 & 574.0 & 0.4089 & 195 \\
\hline
\end{tabular}

The calculation flow chart of improved non sequential Monte Carlo method as shown in Fig. 2:

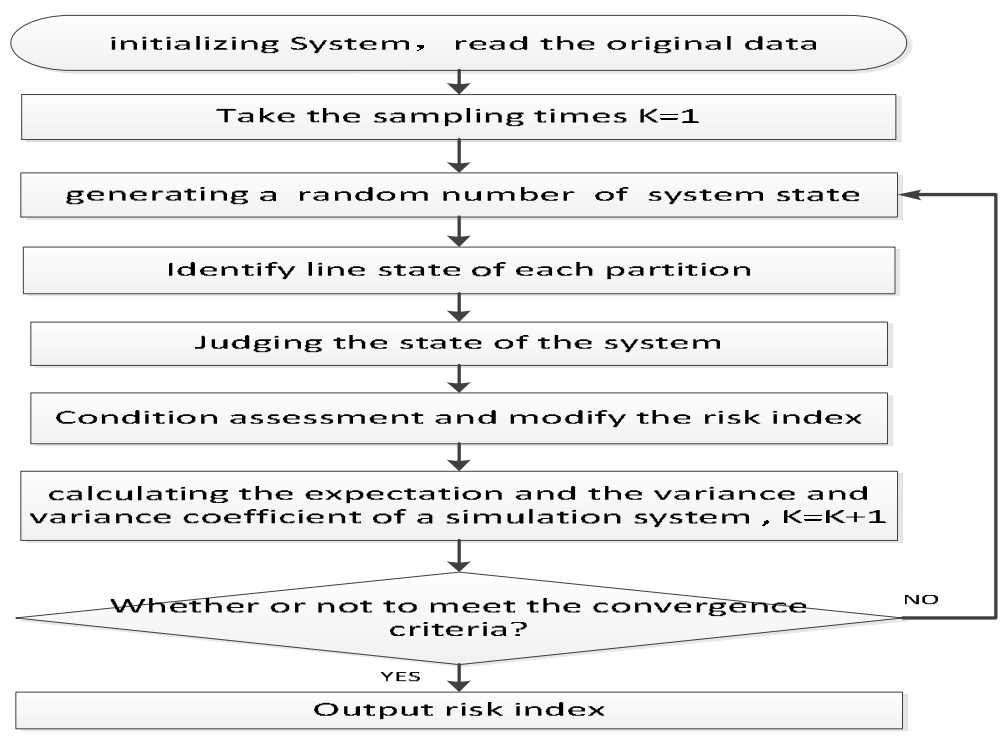

Fig. 2 The calculation flow chart of improved non sequential Monte Carlo method

The theory based on the non-sequential Monte Carlo simulation algorithm of probability, scattered sampling method and analytical judgment were as follows. The risk assessment of power distribution system has been done by using improved and unimproved Monte Carlo method respectively. The calculated risk index were the loss of load probability LOLP and the expected power not supplied value EPNS. The convergence rate of EPNS is slower than rate of LOLP, so the variation coefficient of EPNS has been taken as convergence criterion in order to balance computational accuracy of both in these paper.The assessment results of RBTS-BUS5 ring network structure by two methods as shown in table 3

Table 3 .The risk assessment results of RBTS-BUS5 by Uniform sampling method and scattered sampling method

\begin{tabular}{cccccccc}
\hline \multirow{2}{*}{ Method } & \multirow{2}{*}{$K$} & \multirow{2}{*}{ LOLP } & $V_{L O L P}$ & $\eta_{L O L P}$ & $E P N S$ & $V_{E P N S}$ & $\eta_{E P N S}$ \\
\hline 1 & \multirow{2}{*}{0000} & 0.12689 & 0.00560 & 0.58964 & 3.03904 & 35.91334 & 1.97193 \\
2 & & 0.13227 & 0.10963 & 2.50316 & 3.22738 & 258.10765 & 4.97794 \\
1 & \multirow{2}{*}{20000} & 0.12701 & 0.00560 & 0.58893 & 3.04638 & 35.93139 & 1.96768 \\
2 & & 0.13355 & 0.11044 & 2.48828 & 3.26143 & 260.72031 & 4.95084 \\
1 & \multirow{2}{*}{30000} & 0.12711 & 0.00560 & 0.58878 & 3.04751 & 35.96869 & 1.96797 \\
2 & & 0.13393 & 0.11074 & 2.48471 & 3.26884 & 261.45811 & 4.94661 \\
1 & \multirow{2}{*}{40000} & 0.12721 & 0.00559 & 0.58749 & 3.04582 & 35.87051 & 1.96637 \\
2 & & 0.13396 & 0.11075 & 2.48437 & 3.28076 & 262.42012 & 4.93770 \\
1 & \multirow{2}{*}{50000} & 0.12728 & 0.00559 & 0.58727 & 3.04915 & 35.94385 & 1.96623 \\
2 & & 0.13417 & 0.11099 & 2.48303 & 3.28780 & 262.83584 & 4.93102 \\
\hline
\end{tabular}


Where, method 1 and 2 represent improved and unimproved Monte Carlo method respectively, $K$ is the sampling times, $V_{L O L P}$ and $V_{E P N S}$ mean variances of test functions which correspond to the risk indicators LOLP and EPNS respectively, $\eta_{L O L P}$ and $\eta_{E P N S}$ are the variance coefficient of the corresponding LOLP and EPNS respectively.

\section{Conclusions}

The results of risk assessment in the above examples indicated that method 2 reduces the sampling variance to a certain extent and improve the convergence speed of the Monte Carlo method compared with method 1 for the same sampling number. For instance, the example analysis of RBTS-BUS5 revealed that the variances of test functions ( $V_{L O L P}$ and $V_{E P N S}$ ) calculated by method 1 are 0.00559 and 35.94385 respectively, the variances calculated by method 2 are about 0.11099 and 262.83584 respectively after 50000 times of sampling calculation. The ratio of the variance of Test functions calculated by method 1 and the variance by method 2 were about 5.04\% and $13.68 \%$ respectively. In other words, method 1 has better convergence property compared with methods 2. This can also be verified by size relationship of variance coefficient of two methods. For example, the variances coefficient $\left(\eta_{L O L P}\right.$ and $\left.\eta_{E P N S}\right)$ calculated by method 1 are 0.58964 and 1.97193 respectively, the variances coefficient calculated by method 2 are about 2.50316 and 4.97794 respectively after 10000 sampling calculation. The ratio of the former and the latter were $23.55 \%$ and $39.61 \%$ respectively. What is more, the ratio was $23.65 \%$ and $39.87 \%$ after 50000 times of sampling calculation. Fig. 3 and Fig. 4 described the change law and contrast about $V_{L O L P}$ and $V_{E P N S}$ of the two methods.

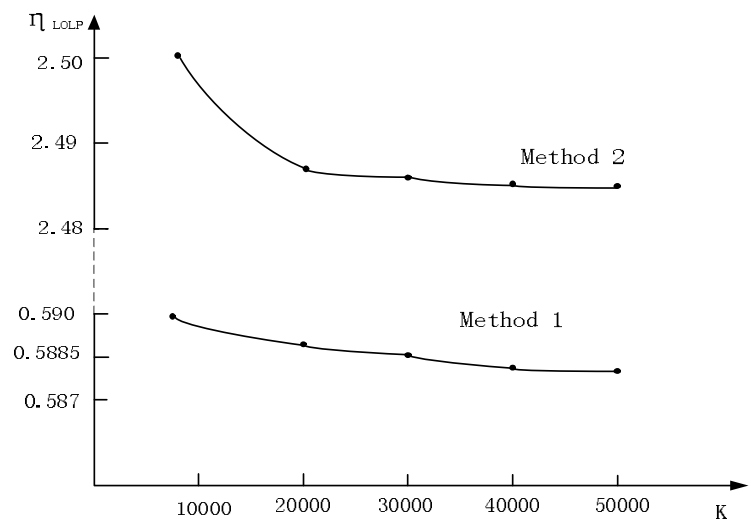

Fig.3 the value of $\eta_{L O L P}$ in different sampling times under the method of 1 and 2

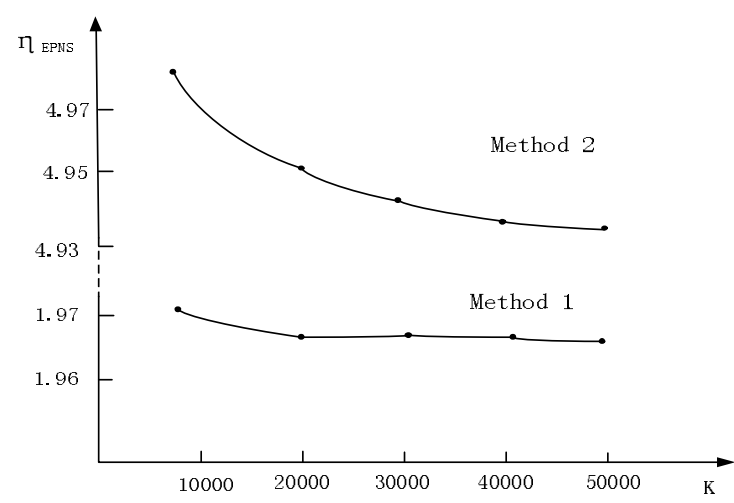

Fig.4 the value of $\eta_{E P N S}$ in different sampling times under the method of 1 and 2

\section{Literature References}

[1] Zaigen Fang. Computer simulation and Monte-Carlo method [M].Beijing: Beijing Institute of Technology Press, 1988.

[2] DMITRII L,ARKADII N,REUVEN Y R.A fast Monte-Carlo method for evaluating reliability indexes[J].IEEE TRANS. on Reliability,1999,48(3):256-261. 
[3] Ming Ding, Shenghu Li. Method for speed up convergence of Monte-Carlo method simulation in reliability calculation[J].Automation of Electric Power Systems,2000,24(12):16-19.

[4] Zhongji Xu. Monte-Carlo method [M].Shanghai: Shanghai Science and Technology Press, 1985.

[5] Billinton R, Li Wenyuan. Reliability Assessment of Electric Power Systems Using Monte Carlo Methods [M]. New York and London: Plenum Press, 1994.

[6] Lizhao Lv, Application of improved non sequential Monte Carlo method in power grid operation mode in risk assessment[D], College of electrical and Information Engineering, Hunan university, 2011.

[7] Huawei Zhang. Investment benefits analysis of feeder automation mode. Journal of QiLu Normal University, 2012, 27 (2), 122-126. 